\title{
The role of joint working between primary and secondary care in supporting people with Type 1 Diabetes
}

Rustam Rea, Consultant in Diabetes and Acute Medicine, Oxford University Hospitals NHS Foundation Trust

Stephen Attwood, GP Bicester Health Centre, GP Lead for North East Locality Oxfordshire CCG

\section{Key points}

- People with Type 1 Diabetes are at high risk of acute and chronic complications due to the long duration of the disease and high glucose variability

- All patients with Type 1 Diabetes should have access to specialist care and the wide range of treatments as described in NICE guidance

- The traditional model of regular attendance at specialist clinics is not effective for all patients with Type 1 Diabetes, particularly those who are regularly attend the emergency department and do not engage with primary or secondary care

- Enablers of joint working include articulating shared outcomes, a shared clinical record, rapid and easy communication between primary and secondary care and other services such as mental health care

\section{Key issues in the care of people with Type 1 Diabetes}

There are over 220,000 people over the age of 17 with Type 1 Diabetes in England and Wales according to the National Diabetes Audit 2016-17 (1). Just over a third (34.4\%) received their eight key care processes and less than a fifth (19.0\%) achieved an HbA1c less than or equal to $58 \mathrm{mmol} / \mathrm{mol}$, a blood pressure of less than or equal to $140 / 80$ and a cholesterol of less than $5 \mathrm{mmol} / \mathrm{L}$ (the triple target). This difficulty in achieving good control of key markers of cardiovascular risk together with the long duration of the disease results in high levels of complications, both acute and chronic (2).

The NICE guidance for the treatment of people with Type 1 Diabetes sets out clearly the expected targets that patients should aim for, accepting that these should be individualised and take into account rates of hypoglycaemia (3). The fact that so few people with Type 1 Diabetes meet these targets is of great concern for patients, those that provide Diabetes services and those that commission the services and are responsible for standards of care.

Many possible reasons have been given for this underachievement and minimal improvement (201516 data showed $18.3 \%$ of people achieving the 3 targets) including poor data capture, poor access to structured education and low levels of uptake of new technology. However a significant factor for an increasing number of patients is the lack of co-ordination between primary and secondary care in performing and or recording the care processes, communicating the results and working together to achieve the patient's goals.

During an acute episode of care patients may be managed by a variety of clinicians including a General Practitioner (GP) in hours, a GP out of hours, an Emergency Department (ED) physician and an Acute Medical Physician. The latter 2 are unlikely to have access to a co-ordinated diabetes record with a clear management plan. This lack of awareness of the patient's overall care can also apply to community providers such as podiatry. The lack of a co-ordinated record across multiple providers results in under recording of care processes and fewer opportunities to act upon them to improve patient outcomes. 
The underlying premise of the split in the National Diabetes Audit reports into people with Type 1 and Type 2 Diabetes is that this would reflect the split in provision of care between secondary care and primary care. However this is often not the case, with many adults with Type 1 Diabetes often receiving their care in primary care and having no contact with specialist Diabetes services (4). This is despite national guidance recommending that all people with Type 1 Diabetes should have a named diabetes consultant and diabetes specialist nurse (5).

Another factor is the sense of ownership of the patient's care. GP practices will vary in the degree of expertise they have in managing people with Type 1 Diabetes. Even within practices this will vary over time with staff turnover. Some practices may feel that responsibility for the overall control of people with Type 1 Diabetes lies with secondary care. When patients don't attend secondary care outpatient appointments and fail to respond to invitations to attend primary care appointments they may fall between the two providers. Indeed the GP 'quality and outcomes framework' which remunerates practices for achieving many of the care processes and the triple targets allows a patient to be excluded from the denominator if they have been invited to an appointment 3 times and not attended. The patient is thus exempted from the practice target calculations but clearly not from the development of complications.

The following three scenarios, based on real people, highlight the need for primary care and secondary care to work more closely together in order to improve the care for people with Type 1 Diabetes.

\section{Scenario 1}

$\mathrm{MrC}$ is a 30 year old man who was diagnosed with Type 1 Diabetes at the age of 20 . He was seen once in secondary care in 2016 and then did not attend any further appointments. His HbA1c over the last few years was between 71 and $115 \mathrm{mmol} / \mathrm{mol}$ and he had multiple admissions with hyperglycaemia and Diabetic Ketoacidosis. The management of his Diabetes was complicated by his increasing use of alcohol and drugs. He was only seen by acute secondary care services (Emergency Department), drug dependency services and very occasionally by his GP.

\section{The role of joint working}

This is an example of where the traditional model of attendance at a secondary care outpatient Diabetes clinic was not effective. People with Type 1 Diabetes may not attend specialist clinics for multiple reasons, some of which may be related to the clinic structure and timing and other reasons will be more specific to the patient. These can include physical and mental health co-morbidities, chaotic lifestyles and being housebound. Sometimes the only services that some patients access are the Emergency Department and drug and alcohol rehabilitation services. These patients often slip under the radar of both primary and secondary care diabetes services through multiple 'Did not attend' appointments and lack of response to written communication.

The solutions to the provision of care for these patients needs to be tailored to their individual needs, however some common themes apply. In the case above, close co-operation and agreed protocols of communication between the Emergency department (ED) and the Diabetes department in the hospital would highlight patients who attend the ED on a frequent basis and do not attend regular diabetes outpatient appointments. Likewise, primary care, as it receives multiple ED discharge summaries, will be in a position to highlight patients who are at high risk of complications to the secondary care Diabetes service. Not only that, but GPs and Practice Nurses are often in a privileged position to understand the broader context within which the patient is trying to manage their diabetes. 
Linking the knowledge of multiple ED attendances, an understanding of the patient's social and psychological context, attendance at other voluntary and rehabilitation services together with the knowledge and expertise of secondary care diabetes professionals is more likely to result in a tailored and effective approach to keeping the patient safe and providing better diabetes care. Often there is no forum to share this knowledge across primary and secondary care and no time set aside to work on possible solutions as the demands on the resources in both primary and secondary care increase. Recognition that setting aside time and effort on both sides to address these difficult scenarios is essential.

\section{Scenario 2}

Mrs A is a 50 year old woman who was diagnosed with Type 1 Diabetes at the age of 20 . She was managed in primary care since her diagnosis and was referred for a possible change in her insulin. Her HbA1c was $55 \mathrm{mmol} / \mathrm{mol}$ and her pre-meal blood glucose values were "pre-breakfast <10, prelunch $10-15$, pre-evening meal $<10$ ". She was having laser treatment and may not be able to drive soon. Her weight had risen to $90 \mathrm{~kg}$ and she was having significant hypoglycaemic episodes once a fortnight. Her current diabetes medication was Lantus 35 units in the evening and Humalog 10 units three times a day.

\section{The role of joint working}

Targets are good and bad. Whether in primary care or secondary care they incentivise certain activity sometimes to the detriment of other aspects of care, which may not be readily measured or seen. Diabetes QoF targets and the triple target in the National Diabetes Audit incentivise lowering $\mathrm{HbA} 1 \mathrm{c}$ to below $58 \mathrm{mmol} / \mathrm{mol}$ without any balancing metrics of either hypoglycaemia or weight gain. NICE guidance calls for individualised target setting but there is no easy way of measuring whether a target is set by the individual or assumed to align with the national target. As a result patients can 'achieve' targets and at the same time suffer significant 'side effects'.

Close working between primary and secondary care can highlight patients being looked after within primary care for whatever reason who are suffering untoward consequences. This collaboration can lead to the appropriate use of technology, such as continuous glucose monitoring or Flash Glucose Monitoring, a greater use of questionnaires such as the Hypo Fear Score and the GOLD score for hypoglycaemia unawareness and a promotion of structured education. In this scenario the patient had not received any structured education for many years and was subsequently referred to a local course.

\section{Scenario 3}

$\mathrm{Mr} \mathrm{B}$ is a 50 year old man who was diagnosed with Type 1 Diabetes at the age of 25 . He has been managed in primary care since his diagnosis and was referred due to erratic blood glucose values in the morning. They were "often 15-16 but if he takes more insulin in the evenings he gets subsequent hypos". He had been on the same insulin regime for 20 years with a "respectable $\mathrm{HbA1c"}(50 \mathrm{mmol} / \mathrm{mol})$.

\section{The role of joint working}

Again, the achievement of national targets for glycaemic control had masked underlying significant problems. Further enquiry into the patient's history revealed significant hypoglycaemia with several recent episodes of severe hypoglycaemia resulting in the patient losing their driving licence. A successful bid by Oxfordshire CCG for Diabetes Transformation Funding has led to the implementation of multi-disciplinary meetings (MDT) at every GP surgery in Oxfordshire. Attendance at these meetings includes a GP, a Practice Nurse, a Diabetes Specialist Nurse from the Community Trust and a Diabetes consultant from the Acute Trust. Each meeting has two main areas of focus. Firstly, increasing each surgery's achievement of the 8 Key Care Processes, triple target and 
structured education and secondly, discussion of key groups of patients. The groups include patients with very high $\mathrm{HbA1c}$, those with a low $\mathrm{HbA1c}$ on insulin or sulphonylureas and patients with a low eGFR. People with Type 1 Diabetes who do not attend secondary care are also discussed with a particular emphasis on hypoglycaemia and structured education. This often leads to a greater awareness of underlying problems as well as therapeutic strategies to address them within the wider patient context.

\section{Addressing the mental health needs of people with Type 1 diabetes}

One of the most challenging aspects of treating people with Type 1 Diabetes is the addition of a significant mental illness. Although the NDA report due out in March 2018 will contain details of the care processes and treatment targets for people with severe mental illness, many people have underlying common mental illness which is managed by the GP or community mental health team or indeed undiagnosed. This can lead to disengagement from treatment and care.

There are a range of models of providing mental health services with IAPT (Improving Access to Psychological Services) accessible from primary care or self-referral and in some areas psychology or psychiatry services available, within secondary care. Clearly close liaison between such services are essential in managing the whole person.

We have developed a model of embedding both IAPT and psychiatry services within a Diabetes MDT meeting (6). Some people with depression and anxiety will benefit from rapid access to IAPT psychological services in groups, while other people with more complex mental health problems will require individual, highly specialist treatment. Seeing psychological input as integral to the discussion of every person with Type 1 Diabetes enables a more holistic approach to patient care, less stigmatisation of mental health problems and greater upskilling of the wider diabetes team.

\section{Lessons}

A joint sense of ownership both of the patients and the outcomes of their care is facilitated by a common contemporaneous shared medical record that can be accessed by all clinicians providing care for the patient. Combining the records of patients across a population enables clinicians from both primary and secondary care to highlight those patients who require additional support, whether education, technology or psychological. It also highlights patients who are not accessing traditional secondary care outpatients or GP care and those in whom the drive to meet national targets is resulting in significant morbidity.

A flexible out-reach approach by secondary care, easily available by phone, e-mail or joint remote consultation (enabled by the GP and consultant screen sharing the patient GP record with skype) allows expert input into the care of people in the community when it is most valuable. Deeper integration of psychological services into the diabetes team helps patients engage with their treatment and provide much needed training and mentoring for the wider Diabetes team.

This new type of service would need to be formed with aligned incentives ideally with an outcomes based contract jointly commissioning services from primary secondary and community care providers. This would be supported by an integrated governance structure and IT to allow population based monitoring of diabetes care. Clear roles for both primary and secondary care will enable greater co-ordination of care and better outcomes for the whole population (Figure 1).

\section{Conclusions}

While the treatment of patients with type 1 diabetes will always remain a challenge, an integrated approach by all providers underpinned by common records, incentives and ownership is likely to produce the best results. Technology will enable some of this to happen and changes in 
commissioning of services across providers will be crucial. Perhaps the most important achievement would be for patients to feel a sense of ownership of their diabetes and that an integrated team is working with them to optimise their care, clinical outcomes and quality of life (Figure 2).

\section{References}

1. National Diabetes Audit 2016-17 Short Report https://digital.nhs.uk/catalogue/PUB30142 (accessed 2.3.18)

2. National Diabetes Audit Complications and Mortality 2015-16 https://digital.nhs.uk/catalogue/PUB30030 (accessed 2.3.18)

3. NICE Guidance for the Diagnosis and Treatment of Type 1 Diabetes https://www.nice.org.uk/guidance/ng17 (accessed 2.3.18)

4. Chiang JL et al. Type 1 Diabetes through the Life Span: A Positionsl Statement of the American Diabetes Association. Diabetes Care 2014: 37: 2034-2054

5. NHS RightCare Pathway: Diabetes https://www.england.nhs.uk/rightcare/products/pathways/diabetes-pathway/ (accessed 2.3.18)

6. Kozlowska $\mathrm{O}$ et al Diabetes care: closing the gap between mental and physical health in primary care. British Journal of General Practice 2017 67(663):471-472

Figure 1

Multiple roles for primary and secondary care in supporting people with Type 1 Diabetes

\begin{tabular}{|l|l|}
\hline \multicolumn{2}{|c|}{ Roles for primary and secondary care in supporting people with Type 1 Diabetes } \\
\hline \multicolumn{1}{|c|}{ Primary Care } & \multicolumn{1}{c|}{ Secondary care } \\
\hline Maintain continuity of relations & Provide structured education \\
\hline Understand the broader context of the patient & $\begin{array}{l}\text { Specialist clinics including insulin pumps and } \\
\text { continuous glucose monitoring }\end{array}$ \\
\hline Re-inforce message of self-care and education & $\begin{array}{l}\text { Remote monitoring of patients through } \\
\text { wearable technology }\end{array}$ \\
\hline $\begin{array}{l}\text { Manage patients who are unable or unwilling to } \\
\text { attend secondary care with specialist guidance }\end{array}$ & Email / telephone advice \\
\hline $\begin{array}{l}\text { Highlight patients who are recurrently admitted } \\
\text { to hospital }\end{array}$ & $\begin{array}{l}\text { Address specific complications - both acute } \\
\text { within hospital and chronic }\end{array}$ \\
\hline & $\begin{array}{l}\text { Access to newer therapies - Islet Cell } \\
\text { Transplantation }\end{array}$ \\
\hline & Recruitment into clinical trials \\
\hline
\end{tabular}

Figure 2

Key aspects to the care of a person with Type 1 Diabetes 
Structured Education - initial and ongoing re-inforcement
Using technology - wearables for patients and rapid communication between all providers

\section{Person with Type}

\section{Diabetes}

Clinical pathway - rapid access to specialist care as inpatient and outpatient
Incentivise self-care within governance structure spanning primary and secondary care 